Full Paper

\title{
Electrochemical Oxidation of Quercetin
}

\author{
Ana Maria Oliveira Brett,* Mariana-Emilia Ghica \\ Departamento de Química, Faculdade de Ciências e Tecnologia, Universidade de Coimbra, 3004-535 Coimbra, Portugal \\ *e-mail: brett@ci.uc.pt
}

Received: December 23, 2002

Final version: February 7, 2003

\begin{abstract}
The mechanism of electrochemical oxidation of quercetin on a glassy carbon electrode has been studied using cyclic, differential pulse and square-wave voltammetry at different $\mathrm{pH}$. It proceeds in a cascade mechanism, related with the two catechol hydroxyl groups and the other three hydroxyl groups which all present electroactivity, and the oxidation is $\mathrm{pH}$ dependent. Quercetin also adsorbs strongly on the electrode surface; and the final oxidation product is not electroactive and blocks the electrode surface. The oxidation of the catechol 3',4'-dihydroxyl electron-donating groups, occurs first, at very low positive potentials, and is a two electron two proton reversible reaction. The hydroxyl group oxidized next was shown to undergo an irreversible oxidation reaction, and this hydroxyl group can form a intermolecular hydrogen bond with the neighboring oxygen. The other two hydroxyl groups also have an electron donating effect and their oxidation is reversible.
\end{abstract}

Keywords: Quercetin, Electrochemistry, Oxidation, Reduction, Adsorption, Radical scavenging activity, pH Dependent antioxidant activity, Polyphenols, Flavonoids, Free radicals

\section{Introduction}

Phenolic compounds are responsible for the brightly colored pigments of many fruits and vegetables, and they protect plants from diseases and ultraviolet light and help prevent damage to seeds until they germinate. Polyphenols are a class of phytochemicals found in high concentrations in wine, tea, grapes and a wide variety of other plants and have been associated with prevention of heart disease and cancer.

The basic features of all polyphenols are the presence of one or more hydroxylated benzene rings. The two main groups of polyphenols are flavonoids and phenolic acids. Flavonoids are benzo- $\gamma$-pyrone derivatives containing several hydroxyl groups attached to ring structures $\mathrm{C}_{6}-\mathrm{C}_{3}-\mathrm{C}_{6}$. They can be subdivided into several groups: flavonols (e.g., quercetin, kaempferol), flavones (e.g., luteolin), flavanols (e.g., catechin), isoflavones (e.g., genistein). Phenolic acids contain two main subgroups: benzoic acid derivatives (e.g., gallic acid) and cinnamic acid derivatives (e.g., caffeic acid, chlorogenic acid) [1]. Flavonoids and related polyphenols generally consist of two benzene rings linked by an oxygencontaining heterocycle (Scheme 1).

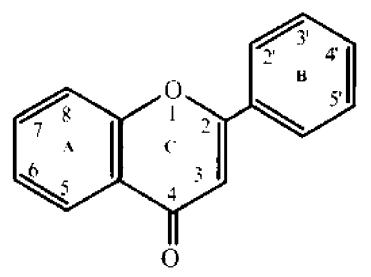

Scheme 1.

Electroanalysis 2003, 15, No. 22
Owing to their polyphenolic nature, flavonoids exhibit strong antioxidant properties. The antioxidant effect of flavonoids can be due to both their radical-scavenging activity and to their metal-chelating properties, of which the former may dominate [2]. In addition to antioxidant and free-radical scavenger properties, flavonoids also exhibit an inhibitory effect on a number of enzymes and potent anticancer effects.

Quercetin is widely distributed in the plant kingdom and is the most abundant of the flavonoid molecules. Quercetin is the aglycone (i.e., minus the sugar group) of a number of other flavonoids, including rutin, quercetrin, isoquercetin, and hyperoside. These molecules have the same structure as quercetin except that they have a specific sugar molecule in place of one of quercetin's hydroxyl groups on the C ring, which dramatically changes the activity of the molecule. Activity comparison studies have identified other flavonoids as often having similar effects as quercetin; but quercetin usually has the greatest activity. Quercetin is the 3, $3^{\prime}, 4^{\prime}, 5,7$, pentahydroxyflavone and the corresponding chemical structure is shown in Scheme 2.

Quercetin appears to cause many beneficial effects on human health, including cardiovascular protection, anticancer activity, anti-ulcer effects, anti-allergy activity, cataract prevention, antiviral activity, and anti-inflammatory effects.

The mechanism of action of flavonoids, as a rule, is as antioxidant, and most of quercetin's effects appear to be due to its antioxidant activity. Quercetin scavenges oxygen radicals [3,4], inhibits xanthine oxidase [5], and inhibits lipid peroxidation in vitro [6]. As another indicator of its antioxidant effects, quercetin inhibits in vitro oxidation of cholesterol low density lipoproteins (LDL), probably by 
<smiles>O=c1c(O)c(-c2ccc(O)c(O)c2)oc2cc(O)cc(O)c12</smiles>

Scheme 2 .

inhibiting LDL oxidation itself, by protecting vitamin $\mathrm{E}$ in LDL from being oxidized or by regenerating oxidized vitamin E [7]. By itself, and paired with ascorbic acid, quercetin reduced the incidence of oxidative damage to neurovascular structures in skin, and inhibited damage to neurons caused by experimental glutathione depletion [8]. Quercetin's anti-inflammatory activity appears to be due to its antioxidant and inhibitory effects on inflammationproducing enzymes (cyclooxygenase, lipoxygenase) and the subsequent inhibition of inflammatory mediators, including leukotrienes and prostaglandins [9, 10]. Inhibition of histamine release by mast cells and basophils [11, 12] also contributes to quercetin's anti-inflammatory activity.

Aldose reductase, the enzyme which catalyzes the conversion of glucose to sorbitol, is especially important in the eye, and plays a part in the formation of diabetic cataracts. Quercetin is a strong inhibitor of human lens aldose reductase [13]. Quercetin exerts antiviral activity against reverse transcriptase of HIV and other retroviruses, and was shown to reduce the infectivity and cellular replication of Herpes simplex virus type 1 , polio-virus type 1 , parainfluenza virus type 3, and respiratory syncytial virus (RSV) [14].

The objective of this study was to investigate the mechanism of oxidation of quercetin by electrochemical techniques, which has not been studied before. The results obtained showed a multi-electron transfer process sequence, $\mathrm{pH}$-dependent, which influences the antioxidant action of quercetin.

\section{Experimental}

\subsection{Reagents}

Quercetin dihydrate $98 \%,\left(3,3^{\prime}, 4^{\prime}, 5,7\right.$, pentahydroxyflavone) was from Sigma-Aldrich, Madrid, Spain, and all the other reagents were Merck analytical grade. All solutions were made up using high-purity water from a Millipore Milli-Q system (resistivity greater than or equal to $18 \mathrm{M} \Omega \mathrm{cm}$ ). Stock solutions of $10^{-3}$ and $5 \times 10^{-5} \mathrm{M}$ quercetin were prepared in ethanol and kept in the refrigerator. These solutions were diluted to the convenient concentration after mixing buffer supporting electrolyte.
Table 1. Supporting electrolytes, $0.2 \mathrm{M}$ ionic strength.

\begin{tabular}{rl}
\hline $\mathrm{pH}$ & Composition \\
\hline 1.6 & $\mathrm{HCl}+\mathrm{KCl}$ \\
3.5 & $\mathrm{HAcO}+\mathrm{NaAcO}$ \\
4.2 & $\mathrm{HAcO}+\mathrm{NaAcO}$ \\
5.3 & $\mathrm{HAcO}+\mathrm{NaAcO}$ \\
5.5 & $\mathrm{NaH}_{2} \mathrm{PO}_{4}+\mathrm{Na}_{2} \mathrm{HPO}_{4}$ \\
7.1 & $\mathrm{NaH}_{2} \mathrm{PO}_{4}+\mathrm{Na}_{2} \mathrm{HPO}_{4}$ \\
7.7 & $\mathrm{NaH}_{2} \mathrm{PO}_{4}+\mathrm{Na}_{2} \mathrm{HPO}_{4}$ \\
9.5 & $\mathrm{NaOH}+\mathrm{Na}_{2} \mathrm{~B}_{2} \mathrm{O}_{7} \cdot 10 \mathrm{H}_{2} \mathrm{O}$ \\
12.1 & $\mathrm{NaOH}+\mathrm{KCl}$ \\
\hline
\end{tabular}

Solutions of buffer supporting electrolyte of ionic strength 0.2 were used in all experiments, Table 1 , and were prepared using analytical grade reagents and purified water from a Millipore Milli-Q system (conductivity $<0.1 \mu \mathrm{S} \mathrm{cm}^{-1}$ ).

\subsection{Apparatus}

The electrochemical experiments were done using an Autolab PGSTAT 10 running with GPES (General Purpose Electrochemical System) version 4.8, software PG (EcoChemie, Utrecht, The Netherlands). Voltammetric curves were recorded at room temperature in a three-electrode system (Cypress System, Inc., USA). The working electrode was a glassy carbon mini-electrode of $1.5 \mathrm{~mm}$ diameter, $\mathrm{Ag} / \mathrm{AgCl}$ (saturated) was used as a reference electrode and platinum wire as a counter electrode. They were all used in a conjunction with an electrochemical cell of $2 \mathrm{~mL}$. The glassy carbon working electrode was cleaned with diamond spray $(25,6$ and $3 \mu \mathrm{m})$. Cyclic voltammetry scan rates were 50 and $100 \mathrm{mV} \mathrm{s}^{-1}$. The differential pulse voltammetry conditions used were: pulse amplitude $50 \mathrm{mV}$, pulse width $70 \mathrm{~ms}$ and scan rate $5 \mathrm{mV} \mathrm{s}^{-1}$. Square-wave voltammetry conditions used were: frequency $50 \mathrm{~Hz}$, amplitude $50 \mathrm{mV}$, and potential increment $2 \mathrm{mV}$ (effective scan rate $100 \mathrm{mV} \mathrm{s}^{-1}$ ).

The $\mathrm{pH}$ measurements were carried out with a CRISON GLP $21 \mathrm{pH}$-meter at room temperature.

\section{Results and Discussion}

It has been shown that the antioxidant activity of flavonoids resides in the aromatic $\mathrm{OH}$ groups [15]. The structure of quercetin presents 5 functional $\mathrm{OH}$ groups, which can undergo oxidation or reduction, and its activity does not reside only in these groups and is enhanced by the carbonyl group, although, generally, the introduction of a carbonyl group in phenol reduces the antioxidant activity.

\subsection{Cyclic Voltammetry}

The oxidation of quercetin studied by cyclic voltammetry at pH 7.7 showed four oxidation peaks, Figure 1, occurring at the potentials of $+0.15,+0.30,+0.60$ and $+0.80 \mathrm{~V}$. These 


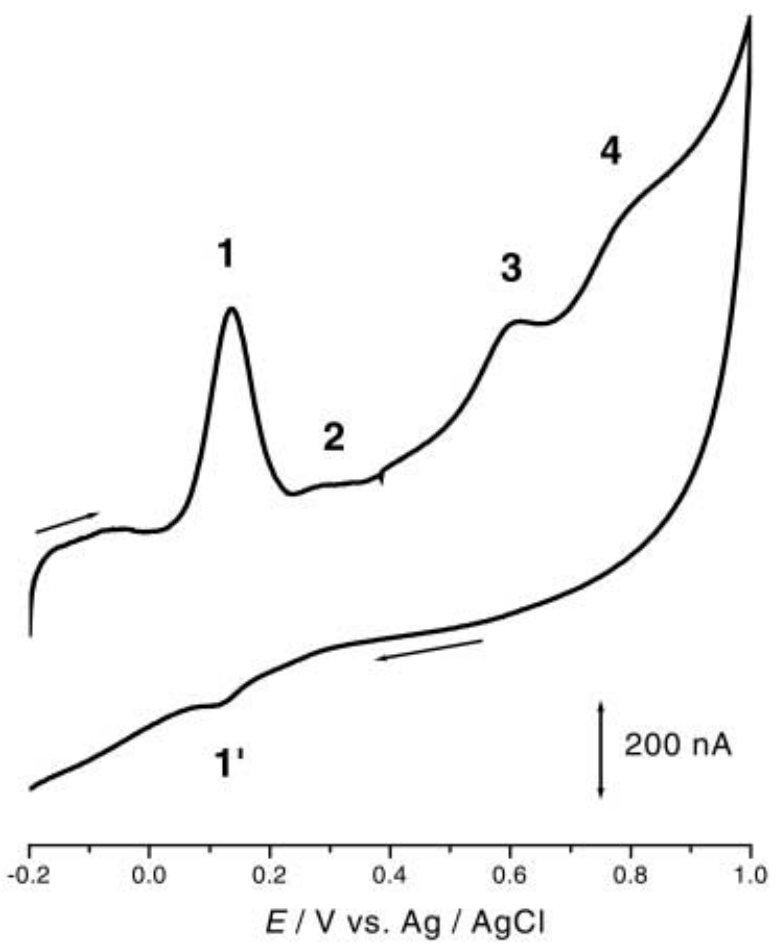

Fig. 1. Cyclic voltammogram of $10^{-5} \mathrm{M}$ quercetin in $\mathrm{pH} 7.7$ phosphate buffer. Scan rate $50 \mathrm{mV} \mathrm{s}^{-1}$.

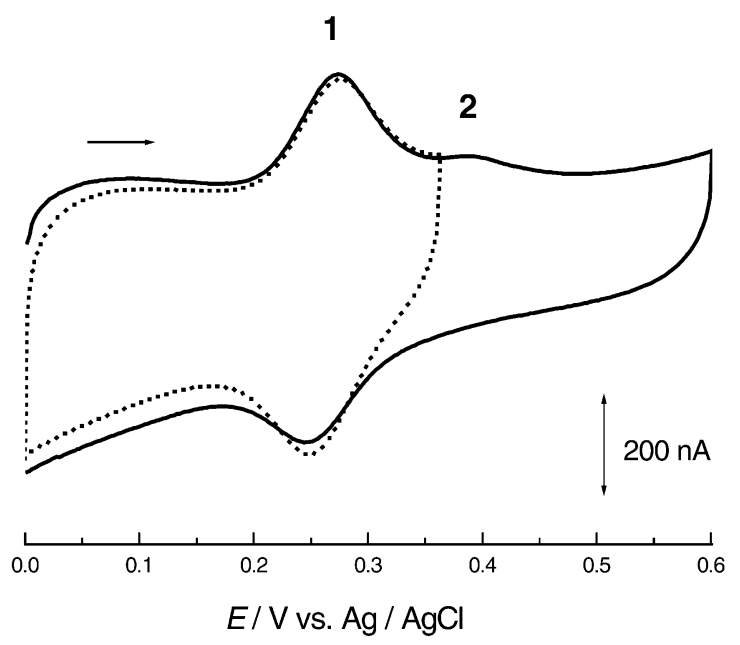

Fig. 2. Cyclic voltammograms of $10^{-5} \mathrm{M}$ quercetin in $\mathrm{pH} 5.5$ phosphate buffer: $(-)$ first scan between 0.0 and $+0.60 \mathrm{~V},(\cdots)$ second scan between 0.0 and $+0.35 \mathrm{mV}$. Scan rate $50 \mathrm{mV} \mathrm{s}^{-1}$.

oxidation peaks are associated with oxidation of the 5 functional $\mathrm{OH}$ groups of quercetin. A reduction peak at about $+0.1 \mathrm{~V}$ could also be seen corresponding to reduction of the oxidation products formed in peak 1 . The reversibility of peak 1 was detected more clearly at $\mathrm{pH} 5.5$ and was confirmed in the second scan by inverting the potential scan just before peak 2, Figure 2. This cyclic voltammogram clearly shows the reversible character of quercetin first electron transfer oxidation reaction. If the scan potential range is extended until peak 4 appears, i.e.,

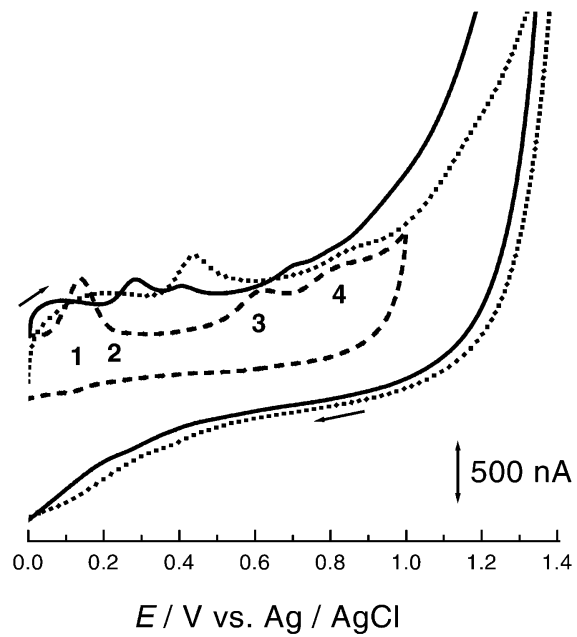

Fig. 3. Cyclic voltammograms of $10^{-5} \mathrm{M}$ quercetin in ( $\cdots$ ) pH 3.5 acetate buffer, (--) pH 5.5 phosphate buffer, pH 7.7 (-) phosphate buffer. Scan rate $100 \mathrm{mV} \mathrm{s}^{-1}$.

$+1.4 \mathrm{~V}$, it is not possible to observe peak $1^{\prime}$ as all the product of the first oxidation of quercetin has reacted in subsequent oxidation reactions, as observed in Figure 3. Moreover, quercetin also adsorbs strongly on the electrode surface and the final oxidation product blocks the electrode surface, shown by the rapid decrease of the quercetin oxidation peak 1 , as evidenced below using differential pulse voltammetry.

\subsection{Differential Pulse Voltammetry}

A differential pulse voltammetry study was performed for a wide $\mathrm{pH}$ range from 1.6 to 12.1 of aqueous buffer supporting electrolyte, Figures 4 and 5 . In this way the dependence of the oxidation of quercetin on $\mathrm{pH}$ was verified. The strong adsorption of its oxidation product, which blocked the electrode surface, was also observed, since quercetin oxidation peak 1 , as well as the others, decreased drastically in the second scan for all $\mathrm{pH}$ values, Figure 4. The main peaks observed for most $\mathrm{pHs}$ were peaks 1 and 2 , whereas peaks 3 and 4 occur only as shoulders and disappeared in alkaline media. The currents of peaks 1 and 2 were smaller in acid and alkaline media and increased at neutral $\mathrm{pH}$, as seen in Figure 5a, the 3D plot of differential pulse voltammograms as a function of $\mathrm{pH}$.

The plot of $E_{\mathrm{p}} \mathrm{vs}$. pH for peaks 1 and 2 shows that protons are involved in the oxidation of quercetin, meaning that during the reaction not only electron but also protons are released from the molecule. The slope of the $E_{\mathrm{p}} \mathrm{vs}$. $\mathrm{pH}$ plot is $65 \mathrm{mV}$ per $\mathrm{pH}$ unit, for peaks 1 and 2 , for $\mathrm{pH}$ lower than 7.7, which corresponds to a mechanism with the same number of protons and electrons involved, and $\mathrm{pH}$ dependent in acid and neutral media. At higher $\mathrm{pH}$ values, between 9 and 12, the values of $E_{\mathrm{p}}$ are independent of $\mathrm{pH}$, so protons no longer participate in the oxidation process, i.e., the reduced form is already chemically deprotonated, and the corresponding $\mathrm{p} K_{\mathrm{a}} \approx 9$ could be found. 

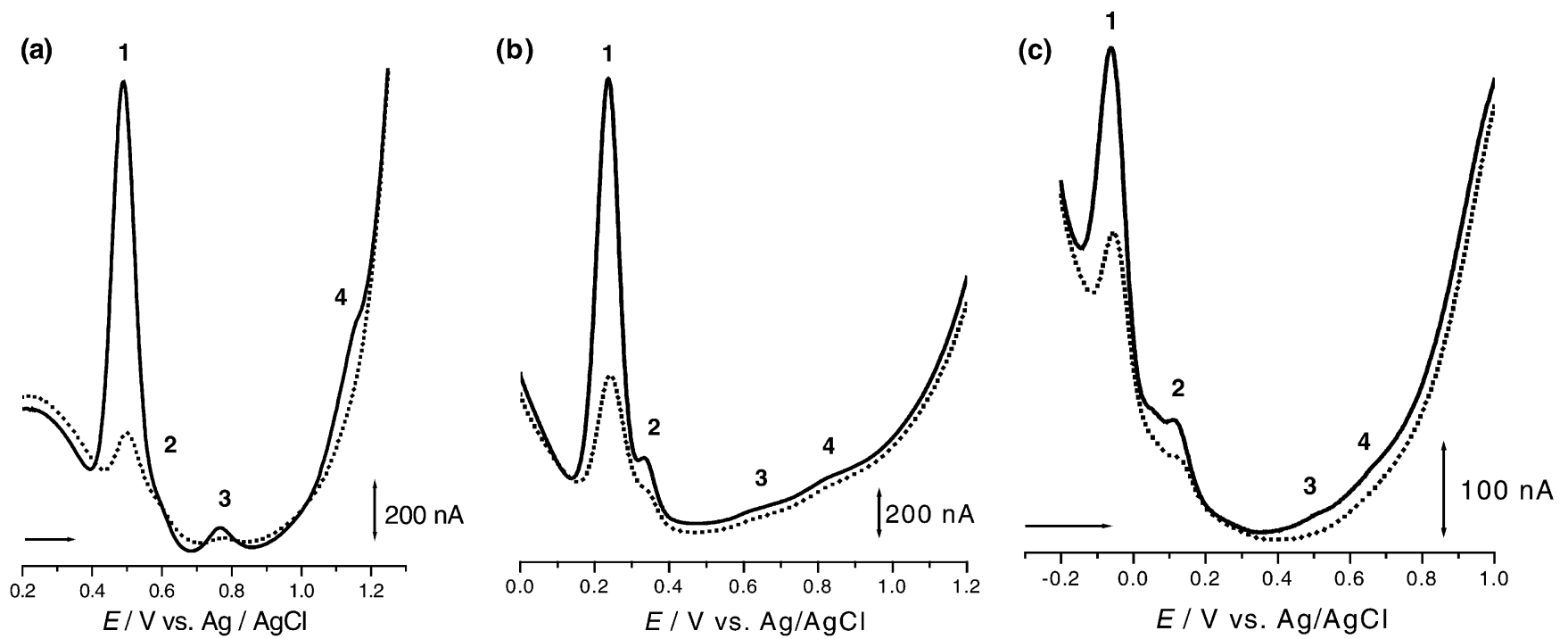

Fig. 4. Differential pulse voltammograms for oxidation of $10^{-5} \mathrm{M}$ quercetin, $(-)$ first and ( $\cdots$ ) second scans, in supporting electrolyte: a) $\mathrm{pH}$ 1.6; b) $\mathrm{pH} 5.5$; and c) $\mathrm{pH}$ 12.1. Scan rate $5 \mathrm{mV} \mathrm{s}^{-1}$.

\subsection{Square-Wave Voltammetry}

The advantages of square-wave voltammetry are greater speed of analysis, lower consumption of electroactive species in relation to differential pulse voltammetry, and reduced problems with blocking of the electrode surface. Square-wave voltammetry showed similar results to differential pulse and cyclic voltammetry, i.e., oxidation peaks $1-4$, large adsorption on the second scan, Figure 6, and increasing current with the $\mathrm{pH}$ in acidic media and decreasing in alkaline media. The curves in square-wave voltammetry were not so well defined because of the faster scan rate used; the effective scan rate used was $100 \mathrm{mV} \mathrm{s}^{-1}$. The square-wave voltammogram which shows more clearly all four peaks corresponding to the electron transfer process was obtained at $\mathrm{pH} 4.1$, Figure 6, where the experimental curves were background-subtracted and base line corrected using the moving average method with a step window of $1 \mathrm{mV}$ included in the GPES software.

The reversibility of the oxidation peaks 1 and 3 of quercetin is clearly shown in Figure 7, where the forward and backward currents are equal and the oxidation and reduction peaks occur at the same potential. Also the first and the second peaks are so close that the latter could not be identified and peak 4, the last oxidation reaction in quercetin, shows reversibility though the peak is very small. A great advantage of the square-wave method is the possibility to see during one scan if the electron transfer reaction is reversible or not. Since the current is sampled in both the positive and the negative-going pulses, peaks corresponding to the oxidation or reduction of the electroactive species at the electrode surface can be obtained in the same experiment. These results compare very well with the results obtained for the same $\mathrm{pH} 1.6$ using differential pulse voltammetry, Figure 4.

\subsection{Quercetin Oxidation Processes}

Previous research showed that the $\mathrm{pH}$-dependent effect on hydroxyflavone antioxidant activity is mainly due to an increased radical scavenging ability of the flavonoids upon their deprotonation. Because deprotonation generally enhances the antioxidant action of the hydroxyflavones and because only the ionization potential (IP), and not the bond dissociation energies (BDE) of the hydroxyflavones, becomes significantly lower upon deprotonation, it can be concluded that electron donation is the dominant mechanism of antioxidant action of hydroxyflavones after deprotonation. Upon deprotonation the radical scavenging capacity increases because electron and not proton donation becomes easier. This implies that not only the ease of radical scavenging but also the mechanism of antioxidant activity may change upon deprotonation, and electron donation may be more important for flavonoid antioxidant action at physiological $\mathrm{pH}[16]$.

Quercetin has two different pharmacophores, the catechol group in ring $\mathrm{B}$ and the three hydroxyl groups in rings $\mathrm{A}$ and C. The activity of the $3 \mathrm{OH}$ groups is enhanced by an electron donating effect of the hydroxyl groups at positions 5 and 7. The hydroxyl groups in ring B are electron-donating and stabilize active intermediates, and the C-3 hydroxyl group can also form intermolecular hydrogen bonds with the oxygen at C-4, also stabilizing active intermediates.

The quercetin oxidation processes proceed in a cascade mechanism and are related with the catechol groups in ring $B$ and the three hydroxyl groups in rings $\mathrm{A}$ and $\mathrm{C}$ which all present electroactivity, and the oxidation potentials are identified with the experimental peaks $1-4$ described previously. The oxidation of the catechol moiety, $3^{\prime}, 4^{\prime}$-dihydroxyl electron-donating groups at ring $\mathrm{B}$, occurs first at very low positive potentials corresponding to peak 1 , and is a two electron two proton reversible reaction (Scheme 3). 
(a)
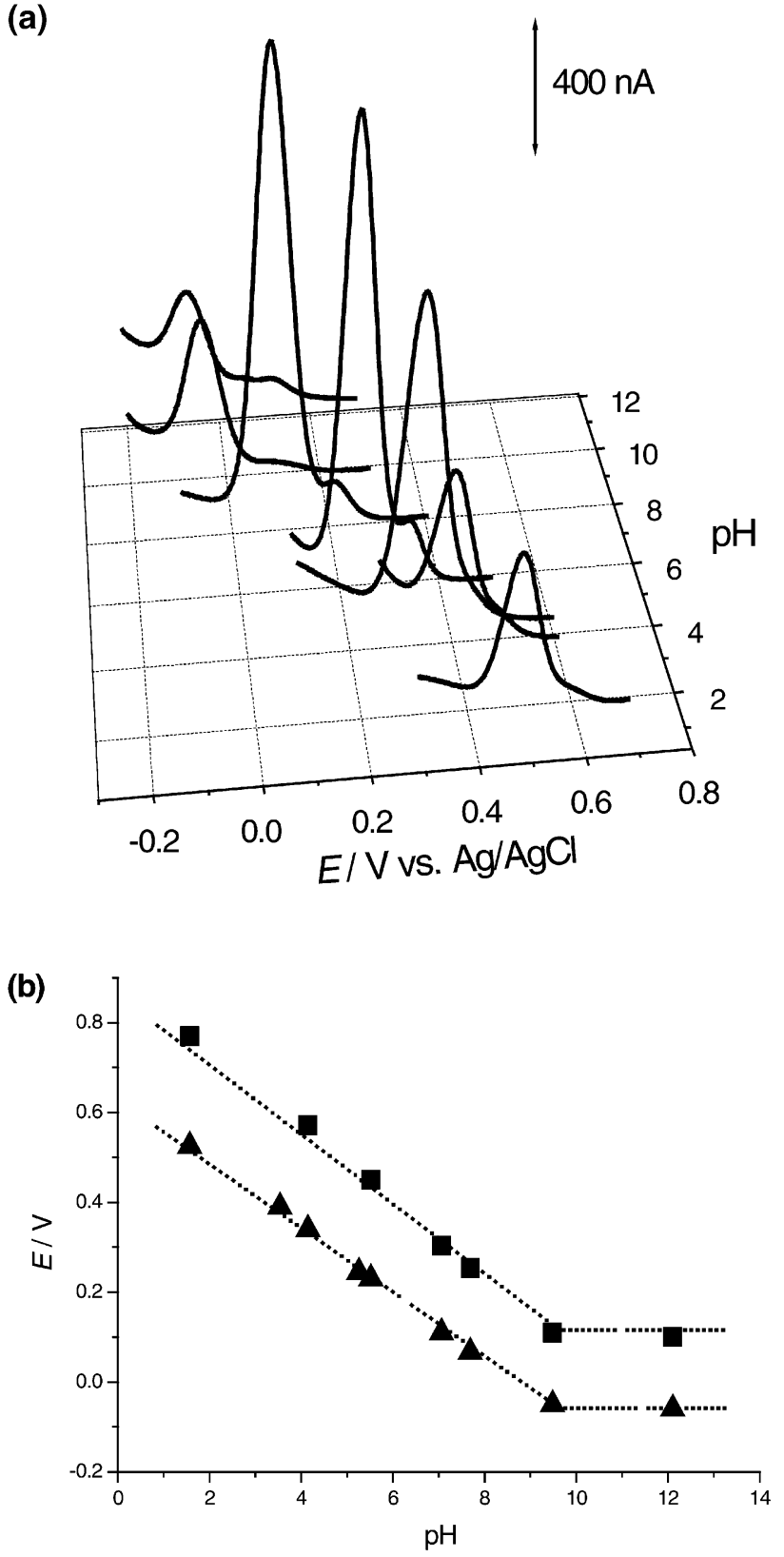

Fig. 5. a) 3D plot of differential pulse voltammograms as a function of $\mathrm{pH}$. Scan rate $5 \mathrm{mV} \mathrm{s}^{-1}$. b) Plot of $E_{\mathrm{p}}$ vs. $\mathrm{pH}$ for $10^{-5} \mathrm{M}$ quercetin in supporting electrolyte: peak $1(\mathbf{})$, and peak $2(\boldsymbol{\Lambda})$.

The hydroxyl group at position 3 at ring $\mathrm{C}$ is oxidized afterwards, corresponding to peak 2 , was shown to undergo an irreversible oxidation reaction, and is a very small peak because the hydroxyl group can also form an intermolecular hydrogen bond with the oxygen at position 4 at ring $\mathrm{C}$. The hydroxyl groups at positions 5 and 7 at ring $\mathrm{A}$ also have an electron donating effect and the oxidation is reversible, as shown by square-wave voltammetry, and occurs for higher positive potentials, corresponding to peaks 3 and 4 .

The current of peak 1 is very high compared with the other three peaks, which is in agreement with higher radical scavenging activity corresponding to the oxidation of the
1

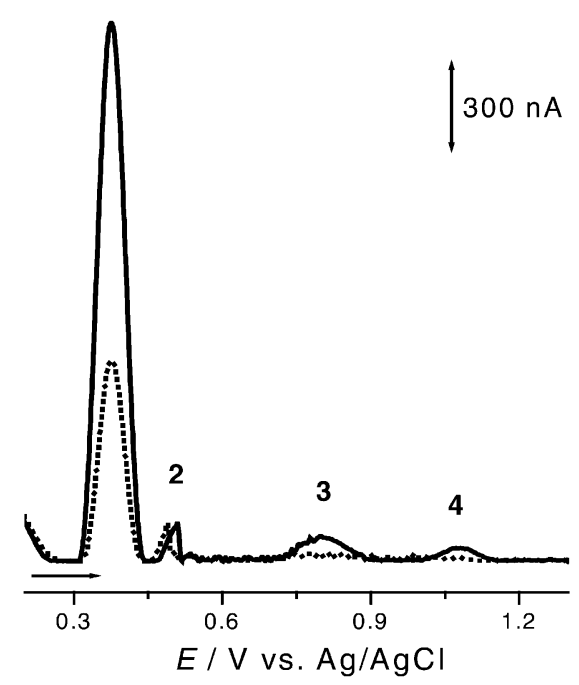

Fig. 6. Square-wave voltammograms for oxidation of $10^{-5} \mathrm{M}$ quercetin in $\mathrm{pH} 4.1$ acetate buffer: $(-)$ first and $(\cdots)$ second scans. Frequency $50 \mathrm{~Hz}$, amplitude $50 \mathrm{mV}$.

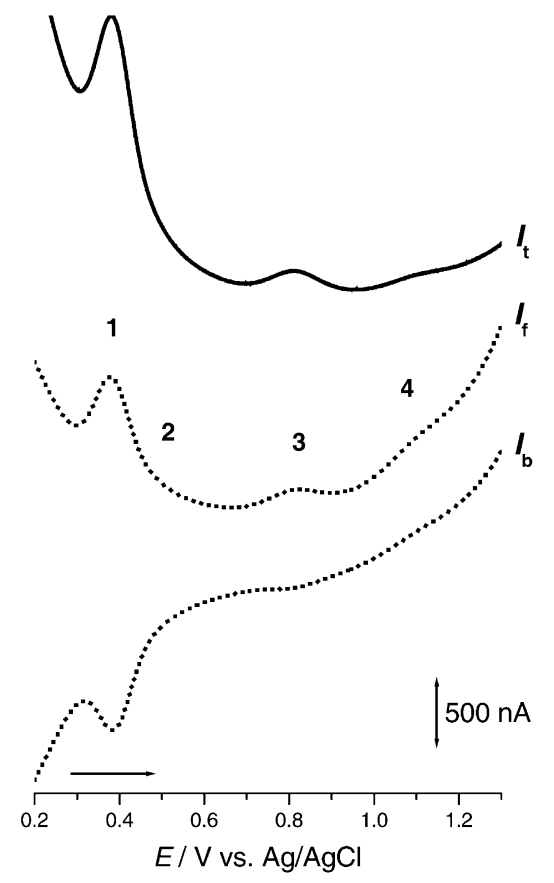

Fig. 7. Square-wave voltammograms for oxidation of $10^{-5} \mathrm{M}$ quercetin in $\mathrm{pH} 1.6$ supporting electrolyte: $I_{\mathrm{t}}$, total current; $I_{\mathrm{f}}$, forward current; $I_{\mathrm{b}}$, backward current. Frequency $50 \mathrm{~Hz}$, amplitude $50 \mathrm{mV}$.

catechol moiety. The oxidation current is strongly dependent on the $\mathrm{pH}$. The oxidation currents are higher at neutral $\mathrm{pH}$ values corresponding to the hydroxyl moiety deprotonation, meaning that after deprotonation electron transfer becomes easier, and the mechanism of radical scavenging antioxidant activity of quercetin in the neutral form is increased. 


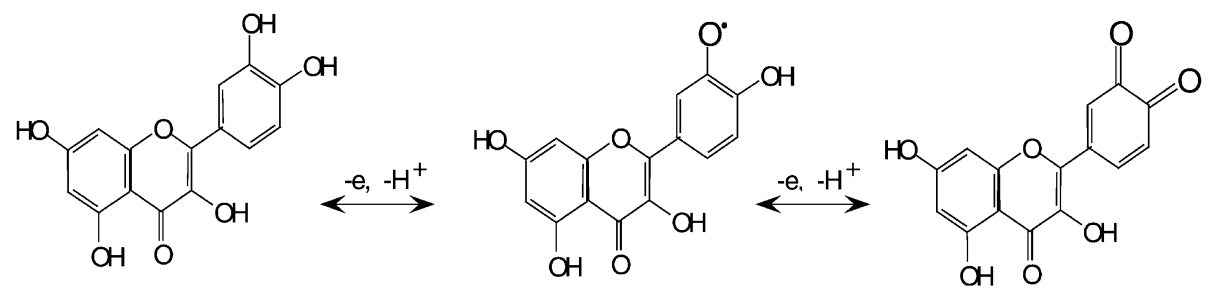

Scheme 3 .

\section{Conclusions}

This study revealed for the first time that the process of electron transfer of quercetin is complex, $\mathrm{pH}$-dependent, most steps are reversible, quercetin is strongly adsorbed on the electrode surface and the final product is not electroactive and blocks the electrode surface. A dependence of peak current on $\mathrm{pH}$ was observed showing a maximum around neutral $\mathrm{pH}$ values and decreasing in acidic and alkaline media. The influence of catechol group deprotonation is related to the electron/proton donating capacity in quercetin and to its radical scavenging antioxidant activity.

\section{Acknowledgements}

Financial support from Fundação para a Ciência e Tecnologia (FCT), POCTI (cofinanced by the European Community Fund FEDER), ICEMS (Research Unit 103) and European Projects QLK3-2000-01311 and HPRN-CT-200200186 are gratefully acknowledged.

\section{References}

[1] M. Filipiak, Analytical Sciences. 2001, 17, 1667.

[2] W. Bors, Handbook on Antioxidants 1996, 406.

[3] A. Saija, M. Scalese, M. Lanza, Free Radical Biology \& Medicine 1995, 19, 481.

[4] A. L. Miller, Alt Med Rev. 1996, 1, 103.

[5] W. S. Chang, Y. J. Lee, F. J. Lu, H. C. Chiang, Anticancer Res. 1993, 13, 2165.

[6] Y. T. Chen, R. L. Zheng, Z. J. Jia, Y. Ju, Free Radical Biology \& Medicine 1990, 9, 19.

[7] C. V. DeWhalley, J. F. Rankin, S. M. Rankin, Biochem Pharmacol. 1990, 39, 1743.

[8] S. D. Skaper, M. Fabris, V. Ferrari, Free Radical Biology \& Medicine 1997, 22, 669.

[9] Della Loggia, E. Ragazzi, A. Tubaro, Pharmacol Res Commun. 1988, 20, S91-S94.

[10] H. P. Kim, I. Mani, V. A. Ziboh, Prostaglandins Leukot Essent. Fatty Acids 1998, 58, 17.

[11] C. C. Fox, E. J. Wolf, A. Kagey-Sobotka, L. M. Lichtenstein, J Allergy Clin Immunol. 1988, 81, 89.

[12] C. Bronner, Y. Landry, Agents Actions 1985, 16, 147.

[13] P. S. Chaudry, J. Cabera, H. R. Juliani, S. D. Varma, Biochem Pharmacol. 1983, 32,1995.

[14] T. N. Kaul, E Jr Middleton, P. L. Ogra, J Med Virol. 1985, 15, 71.

[15] C. G. M. Heijnen, G. R. M. M. Haenen, J. A. J. M. Vekemans, A. Bast, Environ. Toxicol. Pharmacol. 2001, 10, 199.

[16] K. Lemanska, H. Szymusiak, B. Tyrakowska, R. Zielinski, E. M. F. Soffers, I. M. C. M. Rietjens, Free Radical Biology \& Medicine, 2001, 17, 879. 